\title{
The impact of the first wave of the COVID-19 pandemic on the exposure of general surgery trainees to operative procedures
}

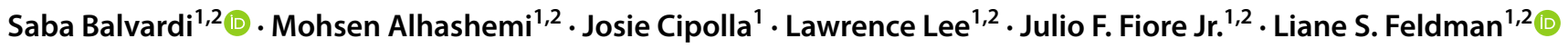

Received: 19 August 2021 / Accepted: 6 December 2021 / Published online: 3 January 2022

(c) The Author(s), under exclusive licence to Springer Science+Business Media, LLC, part of Springer Nature 2021

\begin{abstract}
Introduction During the COVID-19 pandemic, the redeployment of operating room (OR) staff resulted in a significant rampdown of elective surgery. To mitigate the negative effects of the pandemic on surgical education, this study was planned to estimate the impact of the first wave of the pandemic on the participation of general surgery residency and fellowship trainees in operative procedures.

Methods This study is a retrospective review of all adult general surgery procedures performed at 3 sites of an academic health care network. Cases performed during the first wave of the pandemic (March-June 2020) were compared to the same period of the previous year pre-pandemic (March-June 2019). Trainees were categorized as junior (Post-Graduate-Year [PGY] 1-2), senior (PGY3-5), or fellows (PGY6-7). Operating exposure was defined as (1) the total number of cases attended by at least one trainee and (2) total time spent in the OR by all trainees (hours). The impact of the pandemic was estimated as percentage of baseline (2019).

Results During the first wave of the pandemic, a total of 914 cases were performed, compared to 1328 in the pre-pandemic period (69\%). Junior trainees were more affected than senior trainees with reductions in both case volume (68\% versus 78\% of baseline attendance) and time (68\% versus $77 \%$ of baseline operating time). Minimally invasive surgery fellows were most severely affected trainees and colorectal fellows were least affected (14\% and $75 \%$ of baseline cases, respectively). Participation in emergency surgery cases and surgical oncology cases was relatively preserved (87\% and 105\% of baseline, respectively).

Conclusions The first wave of the COVID-19 pandemic reduced operative exposure for general surgery trainees by approximately 30\%. Procedure-specific patterns reflected institutional policies for prioritizing cancer operations and emergency surgeries. These findings may inform the design of remediation activities to mitigate the impact of the pandemic on surgical training.
\end{abstract}

Keywords Operative exposure · Surgical education · COVID-19 pandemic

The first case of COVID-19 in Quebec was diagnosed on February 28th, 2020; as a response, the Quebec government implemented a province-wide order of lockdown and selfisolation on March 13, 2020 [1]. One of the key strategies to expand the healthcare system capacity to treat patients with COVID-19 included a mandated cessation of non-essential

Liane S. Feldman

liane.feldman@mcgill.ca

1 Department of Surgery, McGill University, 1650 Cedar Ave, D6-136, Montreal, QC H3G 1A4, Canada

2 Steinberg-Bernstein Centre for Minimally Invasive Surgery and Innovation, McGill University Health Centre, Montreal, QC, Canada surgical procedures to re-deploy staff and liberate hospital beds [2]. This resulted in a significant decline in access to the operating rooms with only urgent procedures being performed. In addition, fear of being infected by COVID-19 during the first wave of the pandemic may have resulted in a significant decrease in number of patients seeking emergency care [3,4]. Ultimately, this led to a reduction not only in the number of elective surgical procedures being performed, but also emergency procedures. At the same time, measures aiming to limit exposure of health care workers to potentially infected patients needing emergency surgery were implemented, and surgical trainees were redeployed to care for patients with COVID-19. These factors resulted in 
significantly decreased access and participation of surgical trainees in operative cases [5-7].

While there are many adjuncts to facilitate procedural training, such as simulation and on-line curricula [8-11], progressive graduated responsibility in the operating room (OR) is necessary for autonomy and independence of surgical trainees. While longer 5 to 6 years residency training programs may be able to adjust and compensate for a short-term reduction in surgical exposure, trainees in shorter 1-2 year-long fellowships may be more severely impacted. As the pandemic is still ongoing with subsequent waves and new variants appearing, recurrent disruptions in procedural training may have a significant negative impact on the competency of surgical trainees. In addition, this may affect certification decisions in jurisdictions that require attainment of surgical case log benchmarks.

Current available literature reports a reduction in operative exposure for surgical trainees during the pandemic $[7,8$, 12]. However, these studies rely on self-reporting by surgical trainees $[7,8,12]$. An objective measure of the effect of COVID-19 on surgical exposure at each level of training is important to inform the development of tailored short- and long-term remediation plans. The objective of this study is to quantify the impact of the first wave of the COVID-19 pandemic (March 2020-June 2020) on the access of general surgery trainees to operative procedures in comparison to the same period of the previous year pre-pandemic (March 2019-June 2019).

\section{Materials and methods}

\section{Study design}

This study involved the use of an institutional database as well as retrospective chart review to retrieve all emergency and elective operating room procedures performed on General Surgery Clinical Teaching Units (CTUs) at the 3 adult sites of the McGill University Health Center (MUHC). The CTUs include Colorectal/MIS, Bariatrics, Trauma, Hepatobiliary, Transplant, Acute Care Surgery and Surgical Oncology. Data were collected for March to June in both 2019 and 2020. Cases performed during the pandemic period (2020) were compared to the pre-pandemic period (2019). All operations involving adult patients $(\geq 18$ years-old) undergoing elective and emergency surgery performed by a general surgeon attending staff was included in this analysis. The study was approved by the institutional review board (MUHC Research Ethics Board ref. 2021-6972).

General Surgery trainees were defined based on the North American Post-Graduate Year (PGY) System. Trainees were categorized as junior trainee (PGY1-2), senior trainee (PGY3-5) or fellowship trainees (> PGY5) if they were enrolled in a Fellowship Council Accredited program [Advanced GI/Minimally Invasive Surgery (MIS), Bariatric Surgery, Hepatopancreatobiliary Surgery (HPB)] or a Royal College of Physicians and Surgeons of Canada subspecialty training program (Colorectal Surgery).

\section{Measures}

Characteristics of each case were documented. Operating exposure was defined as (1) the total number of cases attended by at least one trainee ("attendance") and (2) the total time spent in the OR by trainees ("hours"). This time is measured from skin incision to skin closure and excludes anesthesia time and the preparatory time. Breakdown of case categories were defined based on the Fellowship Council Specific Fellowship Type Curricula and Program Requirements [13] for fellow trainees and the Royal College of Physicians and Surgeons of Canada Objectives of Training in the Specialty of General Surgery [14].

\section{Statistical analysis}

Continuous variables are presented as mean \pm standard deviation (SD). Categorical variables are presented as $n(\%$ total). Statistical analyses were performed using RStudio (version 1.2.1577; RStudio, Inc., Boston, MA, USA). Analysis of variance (ANOVA) was used to compare the continuous outcomes. The impact of the pandemic on categorical variables was estimated as percentage of baseline (2019).

\section{Results}

While patient characteristics were similar between the two periods, the impact of the pandemic varied by case designation (Table 1). The number of oncology cases remained stable, but these made up a larger proportion of cases (24.3\% pre-pandemic and $37.2 \%$ during the pandemic). The average procedure time was similar between the pre-pandemic and pandemic period $(110.9 \pm 79.9 \mathrm{~min}$ vs. $116.1 \pm 85.5 \mathrm{~min}$, $p=0.167)$. However, the total time in the operating room (including anesthesia time) was significantly higher during the pandemic periods compared to the pre-pandemic baseline $(159.7 \pm 95.9 \mathrm{~min}$ vs. $180.5 \pm 128.5 \mathrm{~min}, p<0.001)$, likely due to the implementation of COVID-19 safety protocols.

The number of residents and fellows assigned to the CTUs was similar in the pre-pandemic and pandemic periods (Table 2). The number of eligible cases attended by at least one trainee was lower in the pandemic period compared to the pre-pandemic period (1328 versus 914 cases, $69 \%$ of baseline). This difference was more pronounced in May and was less so in June (Fig. 1). 
Table 1 Characteristics of operative cases during the prepandemic (March-June 2019) and pandemic (March-June 2020) periods

\begin{tabular}{|c|c|c|c|}
\hline & 2019 & 2020 & $\begin{array}{l}\text { Percent of } \\
\text { baseline } / p \\
\text { value }\end{array}$ \\
\hline \multicolumn{4}{|l|}{ Patient characteristics } \\
\hline Age (years) & $55.3 \pm 17.7$ & $57.6 \pm 15.7$ & $p<0.001$ \\
\hline ASA (> II) & $734(55.3 \%)$ & $515(56.3 \%)$ & $70.2 \%$ \\
\hline \multicolumn{4}{|l|}{ Case designation } \\
\hline Trauma cases & $48(3.6 \%)$ & $34(3.7 \%)$ & $70.8 \%$ \\
\hline Oncology cases & $323(24.3 \%)$ & $340(37.2 \%)$ & $105.3 \%$ \\
\hline Other & $957(72.1 \%)$ & $540(59.1 \%)$ & $56.4 \%$ \\
\hline \multicolumn{4}{|l|}{ Urgency } \\
\hline Emergency & $368(27.7 \%)$ & $320(35.0 \%)$ & $87.0 \%$ \\
\hline Elective & $960(72.7 \%)$ & $594(65.0 \%)$ & $61.9 \%$ \\
\hline \multicolumn{4}{|l|}{ Surgical approach } \\
\hline Laparoscopic & $568(42.8 \%)$ & $360(39.4 \%)$ & $63.4 \%$ \\
\hline Open & $746(56.2 \%)$ & $542(59.3 \%)$ & $72.7 \%$ \\
\hline Conversion & $14(1.1 \%)$ & $12(1.3 \%)$ & $85.7 \%$ \\
\hline \multicolumn{4}{|l|}{ Anesthesia type } \\
\hline Local & $40(3.0 \%)$ & $42(4.6 \%)$ & $105.0 \%$ \\
\hline General & $1257(94.7 \%)$ & $840(91.9 \%)$ & $66.8 \%$ \\
\hline Spinal & $31(2.3 \%)$ & $31(3.4 \%)$ & $100.0 \%$ \\
\hline Total cases & 1328 & 914 & $68.8 \%$ \\
\hline Time in operating room (minutes) & $159.7 \pm 95.9$ & $180.5 \pm 128.5$ & $p<0.001$ \\
\hline Procedure time (minutes) & $110.9 \pm 79.9$ & $116.1 \pm 85.5$ & $p=0.167$ \\
\hline
\end{tabular}

Information is presented as $n$ (\% of total cases) or mean $\pm \mathrm{SD}$

ASA American Society of Anesthesia Score
Table 2 Training level and fellowship designation of trainees assigned to the seven Clinical Teaching Units during the pre-pandemic (March-June 2019) and pandemic (March-June 2020) periods

\begin{tabular}{lrc}
\hline Trainee level & 2019 & 2020 \\
& $n$ & $n$ \\
\hline Junior trainees & 15 & 14 \\
PGY1 & 8 & 7 \\
PGY2 & 7 & 7 \\
Senior trainees & 15 & 17 \\
PGY3 & 5 & 6 \\
PGY4 & 5 & 7 \\
PGY5 & 5 & 4 \\
MIS fellow & 1 & 1 \\
HPB fellow & 2 & 2 \\
Colorectal fellow & 3 & 3 \\
Bariatrics fellow & 2 & 2 \\
Total & 38 & 39 \\
\hline
\end{tabular}

$P G Y$ postgraduate level, $M I S$ minimally invasive surgery, $H P B$ hepatobiliary and pancreas surgery

The impact of the pandemic varied by training level and specialty (Table 3). Junior trainees were more severely affected than senior trainees during the pandemic period with $68 \%$ case attendance compared to the pre-pandemic baseline [ 424 cases (2020) vs. 622 cases (2019)] and 68\% operating time compared to baseline [753 h (2020) vs. $1115 \mathrm{~h}$ (2019)]. Senior trainees attended $73 \%$ of the baseline number of cases [582 (2020) vs. 803 (2019)] and had 77\% of the baseline operating time [1229.4 h (2020) vs. $1604.8 \mathrm{~h}$ (2019)]. Among fellows, the MIS trainee was most severely affected [27.0 h (2020) vs. $155.1 \mathrm{~h}$ (2019), 17.4\% of baseline] while colorectal trainees were least affected $[225.0 \mathrm{~h}$ (2020) vs. $252.7 \mathrm{~h}$ (2019), $75.4 \%$ of baseline]. For all training levels, the decline in time was similar to the decline in number of cases, with the exception of the colorectal fellows who participated in $75.4 \%$ of baseline number of cases but had $89.0 \%$ of the baseline operating time, suggesting an increase in case complexity.

The impact of the pandemic on OR attendance by trainee level and case type is reported in Table 4. For junior trainees, compared to the pre-pandemic period, there were important reductions in exposure to hernia [32 (2020) vs. 117 (2019), $27 \%$ of baseline], cholecystectomy [53 (2020) vs. 85 (2019), $62 \%$ of baseline] and thyroid procedures [8 (2020) vs. 27 (2019), 29.6\% percent of baseline]. However, exposure to breast and melanoma cases increased while there was no change in exposure to appendectomy cases. For senior 


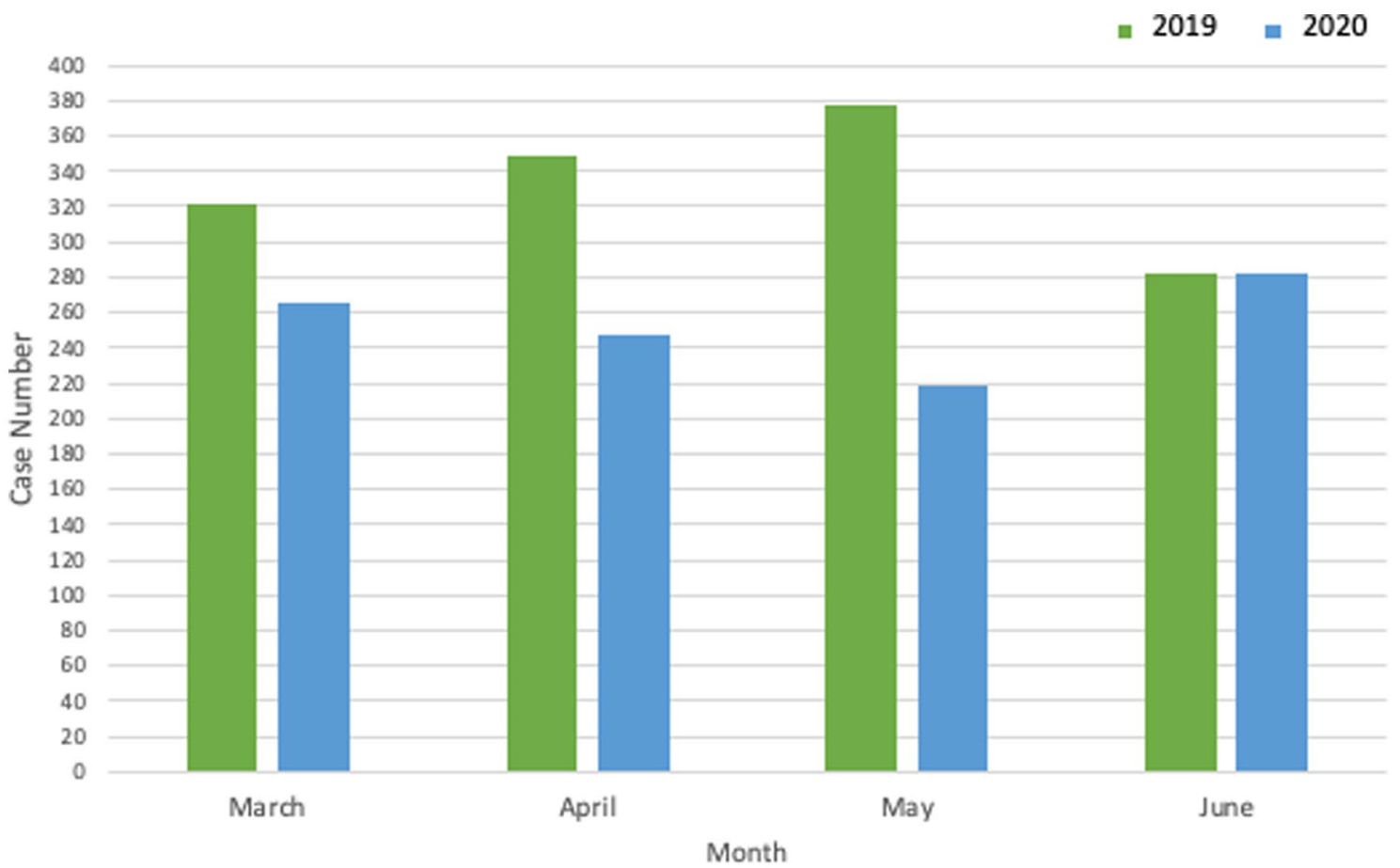

Fig. 1 Trend of General Surgery operations attended by trainees per month

Table 3 Trainee operating room exposure as total cases attended and overall operating time (hours) during the pre-pandemic (March-June 2019) and pandemic (March-June 2020) periods

\begin{tabular}{lllll}
\hline Trainee level & 2019 & 2020 & $\begin{array}{l}\text { Percent of } \\
\text { baseline }\end{array}$ \\
\hline Junior & OR attendance (cases) & 622 & 424 & 68.2 \\
& Overall operating time (hours) & 1115.4 & 752.6 & 67.5 \\
Senior & OR attendance (cases) & 803 & 582 & 72.5 \\
& Overall operating time (hours) & 1604.8 & 1229.4 & 76.6 \\
Fellow & OR attendance (cases) & 582 & 407 & 69.9 \\
& Bariatrics & 133 & 88 & 66.2 \\
& MIS & 85 & 12 & 14.1 \\
& HPB & 132 & 86 & 65.2 \\
Colorectal & 114 & 86 & 75.4 \\
Overall operating time (hours) & 1280.1 & 975.3 & 76.2 \\
Bariatrics & 210.9 & 145.9 & 69.2 \\
MIS & 155.1 & 27.0 & 17.4 \\
HPB & 392.7 & 264.7 & 67.4 \\
Colorectal & 252.7 & 225.0 & 89.0 \\
\hline
\end{tabular}

$O R$ operating room, MIS minimally invasive surgery, $H P B$ hepatobiliary and pancreas surgery

trainees, the most important declines in procedures included stomach and duodenum [10 (2020) vs. 58 (2019), 17.2\% percent of baseline], abdominal wall and hernia [46 (2020) vs. 110 (2019), $41.8 \%$ percent of baseline] and transplant procedures [20 (2020) vs. 42 (2019), 47.6\% percent of baseline].
However, there was increased exposure of senior trainees to breast [60 (2020) vs. 45 (2019), 27.3\% percent of baseline] and colorectal cases [96 (2020) vs. 80 (2019), 120.0\% percent of baseline].

The impact of the pandemic on fellowship case exposure is reported in Table 5. The largest impact was on MIS fellow trainees (participated in 19\% of baseline cases) and bariatrics fellows ( $43 \%$ of baseline cases), with less impact on colorectal (82\% of baseline) and HPB fellows (91\% of baseline). For bariatrics fellowship trainees, malabsorptive procedures were less affected than restrictive procedures ( $77.8 \%$ vs. $22.1 \%$ of baseline). Colorectal fellow trainees had increases exposure to cases involving the rectum $[n=26$ cases (2020) vs. 13 cases (2019), 200\% of baseline] while colon and perianal cases decreased to $57.9 \%$ and $56.2 \%$ of baseline, respectively.

\section{Discussion}

In this study, we identified a $30 \%$ reduction in exposure to operative procedures for general surgery trainees during the first wave of the COVID-19 pandemic in three adult sites of an academic hospital network. All levels of training (i.e., junior, senior, and fellow trainees) experienced a significant decrease in number of operative cases and total time spent in the operating room. Among fellowship trainees, MIS trainees were more severely affected (14\% of pre-pandemic 
Table 4 Procedure types attended by junior and senior trainees during the prepandemic (March-June 2019) and pandemic (March-June 2020) periods

\begin{tabular}{|c|c|c|c|c|c|c|}
\hline & \multicolumn{3}{|c|}{ Junior trainee } & \multicolumn{3}{|c|}{ Senior trainee } \\
\hline & 2019 & 2020 & Percent baseline & 2019 & 2020 & Percent baseline \\
\hline \multicolumn{7}{|l|}{ Abdominal } \\
\hline Abdominal wall \& hernia & 117 & 32 & 27.3 & 110 & 46 & 41.8 \\
\hline Open & 76 & 30 & 39.5 & 72 & 33 & 45.8 \\
\hline Laparoscopy & 41 & 2 & 4.9 & 38 & 13 & 34.2 \\
\hline Cholecystectomy & 85 & 53 & 62.4 & 104 & 80 & 76.9 \\
\hline Liver/advanced biliary & 8 & 8 & 100.0 & 23 & 24 & 95.8 \\
\hline Pancreas & 4 & 7 & 175.0 & 15 & 17 & 113.3 \\
\hline Spleen & 2 & 1 & 50.0 & 6 & 4 & 66.7 \\
\hline \multicolumn{7}{|l|}{ Alimentary } \\
\hline Stomach and duodenum & 11 & 5 & 45.4 & 58 & 10 & 17.2 \\
\hline Small intestine & 27 & 13 & 48.1 & 43 & 42 & 97.7 \\
\hline Colon and rectum & 52 & 36 & 69.2 & 80 & 96 & 120.0 \\
\hline Appendix & 46 & 45 & 97.8 & 65 & 55 & 84.6 \\
\hline Perianal disease & 18 & 12 & 66.7 & 36 & 24 & 66.7 \\
\hline Breast & 77 & 93 & 120.8 & 45 & 60 & 133.3 \\
\hline Lymph node & 4 & 12 & 300.0 & 8 & 7 & 87.5 \\
\hline \multicolumn{7}{|l|}{ Endocrinology } \\
\hline Adrenal & 1 & 1 & 100.0 & 5 & 4 & 80.0 \\
\hline Thyroid & 27 & 8 & 29.6 & 23 & 11 & 47.8 \\
\hline \multicolumn{7}{|l|}{ Skin and soft tissue } \\
\hline Melanoma & 28 & 34 & 121.4 & 16 & 10 & 62.5 \\
\hline Sarcoma & 5 & 4 & 80.0 & 11 & 8 & 72.7 \\
\hline Wound management & 10 & 10 & 100.0 & 15 & 13 & 86.7 \\
\hline Trauma & 15 & 12 & 80.0 & 26 & 30 & 115.4 \\
\hline Exploratory laparoscopy & 7 & 2 & 28.6 & 10 & 1 & 10.0 \\
\hline Exploratory laparotomy & 1 & 4 & 400.0 & 14 & 5 & 35.7 \\
\hline Transplant & 19 & 18 & 94.7 & 42 & 20 & 47.6 \\
\hline Others & 8 & 10 & 80.0 & 16 & 6 & 37.5 \\
\hline
\end{tabular}

cases) and colorectal trainees were the least affected $(75 \%$ of pre-pandemic cases).

While there was a global decrease in operative exposure of all trainees, specific procedures were differentially affected. During the pandemic period, oncology procedures were preserved and there was a higher proportion of emergency surgeries compared to the pre-pandemic baseline. This pattern reflects institutional and governmental prioritization policies [15]. Trauma surgery volumes decreased to $71 \%$ of the pre-pandemic baseline likely related to strict stay-at-home orders in Quebec [1] and the decrease in automobile use and sports.

For junior trainees, there was preserved exposure to procedures that are within their learning objectives within our institution, including oncology procedures (i.e., breast and melanoma) and emergency procedures (i.e., appendectomy and wound management). However, exposure to nononcologic elective procedures for hernia, gallbladder, and perianal diseases was significantly reduced. Senior trainees experienced a similar pattern, with increased or maintained exposure to procedures with oncologic surgeries involving the pancreas, liver, breast, and rectum. However, their exposure to benign gastric (i.e., bariatrics and foregut) and abdominal wall procedures was significantly reduced. There was also evidence of decreased exposure of junior residents to more complex HPB and colon cases. This is consistent with previous literature that emerged at the introduction of work-hour limitations where reduced intraoperative exposure resulted in decreased time spent in observational and decision-making roles (i.e., first assistant and teacher-assistant), with the primary operating role relatively preserved [16]. These findings corroborate the situational realities of the first wave of the pandemic in Quebec, including decreased availability of testing and personal protective equipment [17] that further limited allowed number of persons in the OR. These findings could be used to inform the organization of educational activities including future supplementary rotations to address identified deficiencies.

To address the lack of access to the OR during the first wave of the pandemic, training programs developed and 
Table 5 Index cases attended by fellowship level trainees during the pre-pandemic (March-June 2019) and pandemic (March-June 2020) periods

\begin{tabular}{|c|c|c|c|}
\hline Procedures & 2019 & 2020 & Percent baseline \\
\hline \multicolumn{4}{|l|}{ Bariatrics } \\
\hline Restrictive & 95 & 21 & 22.1 \\
\hline Malabsorptive & 45 & 35 & 77.8 \\
\hline Complications & 10 & 8 & 80.0 \\
\hline Total & 150 & 64 & 42.6 \\
\hline \multicolumn{4}{|l|}{ MIS } \\
\hline Laparoscopic foregut & 7 & 1 & 14.3 \\
\hline Laparoscopic inguinal hernia repair & 29 & 7 & 24.1 \\
\hline Laparoscopic ventral hernia repair & 12 & 1 & 8.3 \\
\hline Laparoscopic solid organ & 10 & 2 & 20.0 \\
\hline Total & 58 & 11 & 19.0 \\
\hline \multicolumn{4}{|l|}{ HPB } \\
\hline Liver & 33 & 25 & 75.8 \\
\hline Pancreas & 14 & 19 & 135.7 \\
\hline Advanced biliary & 3 & 5 & 166.7 \\
\hline Donor hepatectomy ${ }^{a}$ & 5 & 2 & 40.0 \\
\hline Liver transplant* & 12 & 7 & 58.3 \\
\hline Total & 64 & 58 & 90.6 \\
\hline \multicolumn{4}{|l|}{ Colorectal } \\
\hline Colon & 38 & 22 & 57.9 \\
\hline Rectum & 13 & 26 & 200.0 \\
\hline Perianal and incontinence surgeries & 32 & 18 & 56.2 \\
\hline Pouch procedures & 0 & 2 & NA \\
\hline Total & 83 & 68 & 81.9 \\
\hline
\end{tabular}

$M I S$ minimally invasive surgery, $H P B$ hepatobiliary and pancreas surgery

${ }^{a}$ Donor hepatectomy and liver transplant procedures are not part of index cases in hepatobiliary and pancreas fellowship, but they are listed as contributary to complex liver procedure

shared creative strategies including innovative online didactic curriculums, online conferences, and telehealth visits to augment trainees' clinical training [7-12]. Fewer programs reported increasing trainee access to surgical simulators, likely due to the limited access to these simulators at baseline and the decreased access to off-site simulation centers due to campus shutdowns and physical distancing restrictions [10]. However, these measures cannot fully replace the loss of operative experience and operative technical learning among trainees. Using intraoperative video to provide structured feedback and procedural skills teaching could play a role as an adjunct during the COVID-19 pandemic, but this has been underutilized despite strong evidence supporting its role in augmenting trainee technical performance [18-20]. Video-based learning can extend the apprenticeship model to outside of the operating room and play an important role in optimizing learning opportunities during and beyond the COVID-19 pandemic [20].
This study is consistent with others reporting similar decreases in general surgery trainee operative exposure during the pandemic. However, these reports were based on trainee self-reporting through operative logs [8] or national surveys $[7,12]$ which can be subject to reporting bias [21]. The strength of our study is using our institutional OR database and medical records to track trainee presence and involvement in operative cases. However, this study also has several limitations. Our study was based on the three academic hospitals in our health network and does not include the community hospital experience. However, all hospitals in our region were facing the same limitations that imposed limits on residents' operative exposure such as shortage of personal protective equipment and operating room staff redeployment. We were unable to quantify changes in resident role and extent of involvement in the operating room to examine the quality of intraoperative learning during the pandemic. We also could not evaluate the impact of the decreased OR exposure on competence or performance of trainees-case volume alone is not the only marker of proficiency. Furthermore, given the relatively short period of this study (4 months) and the significant role of local institutional and governmental policies in allocating surgical resources during the pandemic, our findings may not be generalizable to other training programs. Also, the rebound response to address the backlog in surgical cases between the subsequent waves of the pandemic will vary significantly across different countries and healthcare systems.

In summary, we have demonstrated that even though there was a global decrease in operative exposure of about $30 \%$ during the first wave of the pandemic, the impact varied by trainee level and fellowship speciality. While junior residents had less exposure to hernia, cholecystectomy, and perianal cases, they had more exposure to oncologic cases. Senior trainees had reduced exposure to benign upper GI cases but more exposure to colorectal cases. For fellowship trainees, the impact of the pandemic was significantly reduced in programs with a high proportion of cancer cases (i.e., colorectal and HPB). These findings are relevant because while longer 5 to 6 years residency training programs may be able to adjust and compensate for a short-term reduction in exposure, 1-year fellowship programs may be more severely affected. While these observed patterns are highly dependent on local policies regarding surgical prioritization, these trends are important to inform mitigation strategies tailored for each level of training.

Funding This study was supported by a training award from Fonds de Recherche du Québec-Santé (Grant No. 288097) to Saba Balvardi. 


\section{Declarations}

Disclosures Saba Balvardi, Mohsen Alhashemi, Josie Cipolla, Lawrence Lee, Julio F. Fiore Jr., Liane S. Feldman have no conflict of interest or financial ties to disclose.

\section{References}

1. Québec GD (2020) Available at https://www.quebec.ca/en/health/ health-issues/a-z/2019-coronavirus/measures-orders-in-councilministerial-orders. Accessed on 8 Jun 2021

2. Association CM (2020) Accessed at Deloitte LLP and affiliated entities at https://www.cma.ca/sites/default/files/pdf/Media-Relea ses/Deloitte-Clearing-the-Backlog.pdf. Accessed on 8 Jun 2021

3. De Filippo O, D'Ascenzo F, Angelini F, Bocchino PP, Conrotto F, Saglietto A et al (2020) Reduced rate of hospital admissions for ACS during Covid-19 outbreak in Northern Italy. N Engl J Med 383(1):88-89

4. Perea Del Pozo E, Aparicio-Sanchez D, Hinojosa Ramirez F, Pareja Ciuro F, Duran Munoz-Cruzado V, Sanchez Arteaga A et al (2020) A prospective cohort study of the impact of covid19 world pandemic on the management of emergency surgical pathology. Br J Surg 107(11):e463-e464

5. Shafi AMA, Atieh AE, Harky A, Sheikh AM, Awad WI (2020) Impact of COVID-19 on cardiac surgical training: our experience in the United Kingdom. J Card Surg 35(8):1954-1957

6. Hourston GJM (2020) The impact of despecialisation and redeployment on surgical training in the midst of the COVID-19 pandemic. Int J Surg 78:1-2

7. Aziz H, James T, Remulla D, Sher L, Genyk Y, Sullivan ME et al (2021) Effect of COVID-19 on surgical training across the United States: a national survey of general surgery residents. J Surg Educ 78(2):431-439

8. Purdy AC, de Virgilio C, Kaji AH, Shields Frey E, Lee-Kong $S$, Inaba K et al (2021) Factors associated with general surgery residents' operative experience during the COVID-19 pandemic. JAMA Surg 156:767

9. Juprasert JM, Gray KD, Moore MD, Obeid L, Peters AW, Fehling $D$ et al (2020) Restructuring of a general surgery residency program in an epicenter of the coronavirus disease 2019 pandemic: lessons from New York City. JAMA Surg 155(9):870-875

10. Chick RC, Clifton GT, Peace KM, Propper BW, Hale DF, Alseidi AA et al (2020) Using technology to maintain the education of residents during the COVID-19 pandemic. J Surg Educ 77(4):729-732

11. White EM, Shaughnessy MP, Esposito AC, Slade MD, Korah M, Yoo PS (2021) Surgical education in the time of COVID: understanding the early response of surgical training programs to the novel coronavirus pandemic. J Surg Educ 78(2):412-421

12. Coyan GN, Aranda-Michel E, Kilic A, Luketich JD, Okusanya O, Chu D et al (2020) The impact of COVID-19 on thoracic surgery residency programs in the US: a program director survey. J Card Surg 35(12):3443-3448

13. Council TF (2020) Accessed at The Fellowship Council at http:// fellowshipcouncil.org/about/program-guidelines/. Accessed on 8 Jun 2021

14. Canada RCoPaSo (2017) Accessed at Royal College of Physicians and Surgeons of Canada at https://www.royalcollege.ca/rcsite/ documents/ibd/general_surgical_oncology_otr_e. Accessed on 6 Jun 2021

15. Bouthillier ME, Lorange M, Legault S, Wade L, Dahine J, Latreille J et al (2021) Prioritizing surgery during the COVID-19 pandemic: the Quebec guidelines. Can J Surg 64(1):E103-E107

16 Kairys JC, McGuire K, Crawford AG, Yeo CJ (2008) Cumulative operative experience is decreasing during general surgery residency: a worrisome trend for surgical trainees? J Am Coll Surg 206(5):804-811

17. Dyer E (2020) The great PPE panic: how the pandemic caught Canada with its stockpiles down. CBC News, Toronto

18. Trehan A, Barnett-Vanes A, Carty MJ, McCulloch P, Maruthappu M (2015) The impact of feedback of intraoperative technical performance in surgery: a systematic review. BMJ Open 5(6):e006759

19. Hodges NJ, Chua R, Franks IM (2003) The role of video in facilitating perception and action of a novel coordination movement. $\mathrm{J}$ Mot Behav 35(3):247-260

20. Green JL, Suresh V, Bittar P, Ledbetter L, Mithani SK, Allori A (2019) The utilization of video technology in surgical education: a systematic review. J Surg Res 235:171-180

21. Veldenz HC, Dennis JW, Dovgan PS (2001) Quality control of resident operative experience: compliance with RRC criteria. J Surg Res 98(2):81-84

Publisher's Note Springer Nature remains neutral with regard to jurisdictional claims in published maps and institutional affiliations. 